\title{
Validation of "Signs of Inflammation in Children that Kill" (SICK) score for immediate non-invasive assessment of severity of illness
}

\author{
Manoj A Gupta', Anjan Chakrabarty22, Ruth Halstead², Mohit Sahni1', Jayanti Rangasami2, Ashish Puliyel3, \\ Vishnubhatla Sreenivas', David A Green² and Jacob M Puliyel*1
}

\begin{abstract}
Objective: To validate the SICK scoring system's ability to differentiate between individuals with higher and lower probabilities of death

Method: We performed a one year two-centre prospective evaluation of all children aged between one month and 12 years referred to the Paediatric team at St Stephens Hospital in Delhi and admitted to the Paediatric Department at West Middlesex University Hospital in London. We calculated SICK scores at presentation and correlated them with subsequent in-hospital mortality. We used discrimination by areas under receiver operating characteristic (ROC) curves to measure performance.
\end{abstract}

Results: We prospectively evaluated 3895 children in Delhi and 1473 children in London. The areas under the ROC curves were $84.8 \%$ in Delhi, $81.0 \%$ in London and $84.1 \%$ (95\% Cl $77.4-90.8 \%)$ for combined data. Hosmer-Lemeshow goodness of fit for the combined data was good (Hosmer-Lemeshow Chi-square $=2.13(p=0.345)$.

Conclusions: We propose the SICK score as a useful triage tool at initial presentation and highlight its particular suitability for resource poor settings.

\section{Introduction}

The Early Warning Score [1] and Modified Early Warning Score [2] use physiological parameters to identify high risk patients on adult general wards. However they are not weighted and are based on the number of abnormal parameters. Disease specific early warning scores (e.g. CURB65 for pneumonia) have also been used in adults [3] but less so in children. The first physiological scoring system for children was the Physiology Stability Index (PSI), which derived a subjective score from the worst of 34 values from routinely measured variables over the first day on the Paediatric Intensive Care Unit (PICU) [4]. The PRISM score [5] evolved from it as a simplification with only 14 variables but still used laboratory results and so was cost and labor intensive and not assessable on presentation. The problem of lead-time bias was circumvented by the Pediatric Index of Mortality (PIM, then

\footnotetext{
*Correspondence: puliyel@gmail.com

1 Department of Pediatrics, St Stephens Hospital, Delhi, India Full list of author information is available at the end of the article
}

PIM2), which is calculated directly on admission to PICU [6]. The World Health Organization has developed emergency triage, assessment and treatment (ETAT) guidelines for use in developing countries [7] but a drawback is that these require a specific training program before implementation.

Recently Thompson and colleagues have demonstrated that vital signs can identify sick children in pediatric emergency care with comparable sensitivity to more complex triage systems [8]. They however did not develop a scoring system for use in triage. The "Signs of Inflammation in Children that Kill" (SICK) score was developed in a search for a practical triage tool for resource poor settings. Here we validate it in the context of emergency triage. The score, which evaluates the expected risk of mortality, utilizes the abnormal physical variables of the Systemic Inflammatory Response Syndrome [9] and its continuum - the Multiple Organ Dysfunction Syndrome. Its seven parameters are heart rate, respiratory rate, systolic blood pressure, temperature, 
blood oxygen saturation $\left(\mathrm{SpO}_{2}\right)$, capillary refill time (CRT), and conscious level. It also takes age into account. A development study [10] of 1099 consecutive children admitted to the Paediatric Department at St Stephens Hospital (SSH) in Delhi (comprising a ward and PICU) gave regression coefficients (logs of the odds ratio of death) which are used as weightages. A previous validation study [11] at SSH was limited to 125 PICU admissions. The study we report here is a larger two-centre validation study. The objective was to validate the SICK scoring system's ability to differentiate between individuals with higher and lower probabilities of death.

\section{Methods}

This was a prospective study from 15 November 2005 to 15 November 2006 at SSH and 6 February 2006 to 6 February 2007 at West Middlesex University Hospital (WMUH).

WMUH is a Secondary Care teaching hospital associated with the Imperial College School of Medicine. It has 400 beds and serves a population of approximately 330 000. The Paediatric Department includes a 20-bedded inpatient ward and an 8-bedded day unit but does not have a PICU. SSH is a 600-bed non-governmental hospital, which functions as a tertiary care referral centre. The Paediatric Department includes a 40-bedded inpatient ward plus a 7 bed PICU with three ventilators.

We obtained ethical approval from the SSH research committee in India and the Ealing and West London Mental Health Trust Local Research Ethics Committee in UK (REC approval reference number 05/Q0410/75). Children attending the Emergency department at SSH are first seen by an ED physician, and then referred to the Paediatric team if needed. At SSH this referral was the entry point to the study, whereas at WMUH admission or transfer to neighboring PICUs was the entry point. All patients aged between one month and 12 years (i.e. from the $29^{\text {th }}$ day to the $13^{\text {th }}$ birthday) were consecutively enrolled into the study. This represented a sample of one year's activity. At SSH we excluded patients from the analysis who left against medical advice or were referred to other hospitals (making outcome uncertain).

We evaluated SICK score parameters at entry. We measured axillary temperatures by digital electric thermometry at SSH (Dr. Morepen Digiclassic, Switzerland) and tympanic auricular temperatures at WMUH ("Genius First Time"-Infra red tympanic thermometer). We measured CRT on the sternum or a digit at the level of heart after applying blanching pressure for 5 seconds. We measured conscious level using the AVPU score ("Alert", "Responding to Voice", "Responding to Pain only" or "Unresponsive"). We measured $\mathrm{SpO}_{2}$ with a saturation monitor applied to the skin, usually on a finger (Larsen and Toubro Medical Stellar India at SSH; Phillips Intelli Vue MP 70 or Ohmeda Biox 3740 Pulse Oximeter at WMUH). At SSH we measured blood pressure using a sphygmomanometer cuff covering over $75 \%$ of the length of the upper arm (Larsen and Toubro Medical Stellar India) in all cases. At WMUH we measured blood pressure using an electronic blood pressure monitor (Dinamap - Critikon or Vital Signs Monitor 8100) in selective cases with the combination of increased CRT and decreased conscious level. In patients who were well with normal conscious level and CRT we did not measure BP for pragmatic reasons, on the assumption that hypotension is a late and preterminal sign in paediatric shock [12]. Doctors recorded CRT measurements and AVPU scores. Nurses measured all other parameters. The parameters used were part of the routine examination of children brought to the hospital for evaluation and so consent was not obtained for using the data. No special training of staff was required.

Abnormal ranges were taken as: heart rate $>160 / \mathrm{min}$ ute (infant), $>150 /$ minute (child); respiratory rate $>60 /$ minute (infant), $>50 /$ minute (child); systolic blood pressure $<65$ (infant), $<75$ (child); temperature $\left(>38^{\circ} \mathrm{C}\right.$, $<36^{\circ} \mathrm{C}$ ); $\mathrm{SpO}_{2}(<90 \%)$; CRT ( $\geq 3$ seconds); and conscious level (all states of consciousness except "Alert"). The variables were treated as binomial variables and classified as normal/abnormal.

The weightages taken from the development study [11] were: heart rate (0.2); respiratory rate (0.4); systolic blood pressure (1.2); temperature (1.2); $\mathrm{SpO}_{2}$ (1.4); CRT (1.2); conscious level (2.0); age bands $<1$ year (1.0), 1-5 years $(0.3)$ and $>5$ years $(0.0)$. The range of possible scores was therefore 0 to 8.6 .

We calculated SICK scores by adding the weightage of each abnormal variable using custom-made software 'SICK score calculator' http://jacob.puliyel.com/sick.php. The software defaulted unmeasured parameters as normal. SICK score was not used to make clinical decisions. We followed all patients to discharge. We measured outcome as survival to discharge home or death.

Logistic regression was applied to the outcome using the calculated SICK score as the predictor. We tested the predictive ability of the SICK score by looking at the area under the receiver operating characteristic (ROC) curve. The fitted model was also assessed for its goodness of fit by applying the Hosmer-Lemeshow chi-square test, based on equally sized groups (analogous to $\mathrm{C}$ statistic).

\section{Results}

At SSH, 4116 patients qualified for inclusion. Of these 108 left against medical advice and 113 transferred to another hospital. Thus, we included 3895 children in the analysis. Fifty eight children died. 
At WMUH, 1473 patients were admitted during the study period and we excluded none. Five children died.

The age distributions were similar in the two centers. (Age bands $<1,1-2,2-5,5-10$ and 10-12 years accounted for $35 \%$ versus $31 \%$, $16 \%$ versus $20 \%$, $23 \%$ versus $27 \%, 19 \%$ versus $18 \%$ and $6 \%$ versus $3 \%$ in Delhi and London respectively).

Tables 1 and 2 show observed versus expected mortalities at ascending levels of SICK score in Delhi and London respectively. Distribution of SICK scores between the two populations is significantly different $(\mathrm{p}<0.001)$. Table 3 shows the fitted model for the combined data to assess for goodness of fit by applying the Hosmer-Lemeshow chi-square test. The differences between the observed and expected number of deaths and survivals were statistically not significant in the 4 risk strata. Hosmer-Lemeshow Chi-Square with 2 degrees of freedom was $2.13(\mathrm{p}=0.345)$.

The scoring system performed equally well in both centers. The areas under the ROC were $84.8 \%$ (95\% CI: $78.2 \%$ - 91.5\%) in Delhi, $81.0 \%$ (95\% CI: 45.4\% - 100.0\%) in London and $84.1 \%$ (95\% Confidence Intervals $77.4-90.8 \%$ ) for the combined data set (figure 1). Figure 2 shows the expected and observed mortality (per 1000 in each score category) increasing with SICK score.

\section{Discussion}

An important advantage for clinical practice is that the SICK score can be generated immediately on presenta- tion. Mortality was higher in India than in the UK for any given SICK score (except 6 - 6.9, which was represented by a single patient in the UK). This would be expected and explained by differences in individual health conditions (e.g. nutritional status) and locally available resources.

The calibration of a model evaluates the degree of correspondence between the estimated probabilities of mortality it produces and the actual mortality experience of the patients. It can be statistically evaluated using "goodness-of-fit" tests [13]. Despite the difference in mortality, the calibration of the SICK score (comparing observed and expected mortality at different grades of severity) at the two centers was good. Moreover, our objective was to differentiate between individuals with higher or lower probabilities of death, for which the desired test quality is a good discriminatory capacity (area under ROC curve). The area under ROC curve of $84.1 \%$ for combined data confirmed a good correlation of outcome with SICK score. Furthermore, the areas under ROC curves were consistent with those seen in the development cohort (89\%) [9] and the first validation study (76\%) [10]. These findings imply a high probability of external validity and generalisability. Its performance in identifying sicker patients lends it to use in emergency triage.

Severity-of-illness scores have been developed for the purposes of comparative audit (comparing actual with expected outcomes over different units), evaluative research (adjusting for differences in case mix or as an aid

Table 1: Observed versus expected mortalities at ascending levels of SICK score (Delhi)

\begin{tabular}{|c|c|c|c|c|}
\hline \multirow{3}{*}{$\begin{array}{l}\text { SICK } \\
\text { Score }\end{array}$} & \multicolumn{4}{|l|}{ Delhi (SSH) } \\
\hline & \multicolumn{2}{|l|}{ Died } & \multicolumn{2}{|l|}{ Survived } \\
\hline & Observed & Expected & Observed & Expected \\
\hline 0 & 4 & 1.9 & 805 & 807.0 \\
\hline$<1$ & 2 & 3.5 & 1031 & 1029.5 \\
\hline $1-1.9$ & 8 & 13.0 & 1567 & 1562.0 \\
\hline $2-2.9$ & 13 & 8.2 & 334 & 338.8 \\
\hline $3-3.9$ & 3 & 3.8 & 60 & 59.2 \\
\hline $4-4.9$ & 6 & 3.7 & 19 & 21.3 \\
\hline$\geq 5$ & 22 & 23.8 & 21 & 19.2 \\
\hline Total & 58 & 57.9 & 3837 & 3837.0 \\
\hline
\end{tabular}


Table 2: Observed versus expected mortalities at ascending levels of SICK score (London)

\begin{tabular}{|c|c|c|c|c|}
\hline \multirow{3}{*}{$\begin{array}{l}\text { SICK } \\
\text { Score }\end{array}$} & \multicolumn{4}{|c|}{ London (WMUH) } \\
\hline & \multicolumn{2}{|l|}{ Died } & \multicolumn{2}{|l|}{ Survived } \\
\hline & Observed & Expected & Observed & Expected \\
\hline 0 & 1 & 0.0 & 246 & 247.0 \\
\hline$<1$ & 0 & 0.1 & 394 & 393.9 \\
\hline $1-1.9$ & 0 & 0.6 & 573 & 572.4 \\
\hline $2-2.9$ & 0 & 0.8 & 193 & 192.2 \\
\hline $3-3.9$ & 1 & 0.9 & 47 & 47.1 \\
\hline $4-4.9$ & 2 & 1.4 & 13 & 13.6 \\
\hline$\geq 5$ & 1 & 1.1 & 2 & 1.9 \\
\hline Total & 5 & 4.9 & 1468 & 1468 \\
\hline
\end{tabular}

to stratification in randomised control trials) and clinical management of individual patients (as a triage tool or as a clinical shorthand to rapidly convey patient information). However, the latter purpose has been addressed by very few paediatric scoring systems. An ideal triage scoring system must be available immediately on presentation and the score must accurately describe the severity of illness. The overall goals of triage are to determine if a patient is appropriate for a given level of care and to ensure that hospital resources are utilized effectively.

We measured temperatures in the axilla at $\mathrm{SSH}$ and tympanically at WMUH because this was the standard practice in the two units at the time. Studies have shown that axillary temperature is lower than core temperature: a systematic review of studies found a pooled mean temperature difference of $0.85^{\circ} \mathrm{C}$ for digital electric thermometers [14]. This difference would tend to skew the data towards a relative under diagnosis of fever and over diagnosis of hypothermia at SSH compared to WMUH (although the SICK score would be the same for each category). We felt that for practical purposes this did not make an important difference to score distributions.

We used normal values as default readings for missing values. Consequent misclassifications would spuriously lower the observed sensitivity of the score and would

Table 3: The fitted model for the combined data looking for 'goodness of fit' applying the Hosmer-Lemeshow chi-square test

\begin{tabular}{|c|c|c|c|c|c|c|c|}
\hline \multirow[b]{2}{*}{ Group } & \multirow[b]{2}{*}{ Probability } & \multicolumn{2}{|c|}{ Died } & \multicolumn{2}{|c|}{ Alive } & & \multirow{2}{*}{$\begin{array}{c}\text { Total } \\
\text { Chi- } \\
\text { square* } \\
\text { (p value) }\end{array}$} \\
\hline & & Observed & Expected & Observed & Expected & & \\
\hline 1 & 0.0023 & 6 & 4.4 & 2238 & 2239.6 & 2244 & $2.13(0.345)$ \\
\hline 2 & 0.0047 & 6 & 5.3 & 1223 & 1223.7 & 1229 & \\
\hline 3 & 0.0078 & 3 & 6.0 & 885 & 882.0 & 888 & \\
\hline 4 & 0.8863 & 48 & 47.2 & 959 & 959.8 & 1007 & \\
\hline
\end{tabular}

*Hosmer-Lemeshow Chi-Square with 2 degrees of freedom 


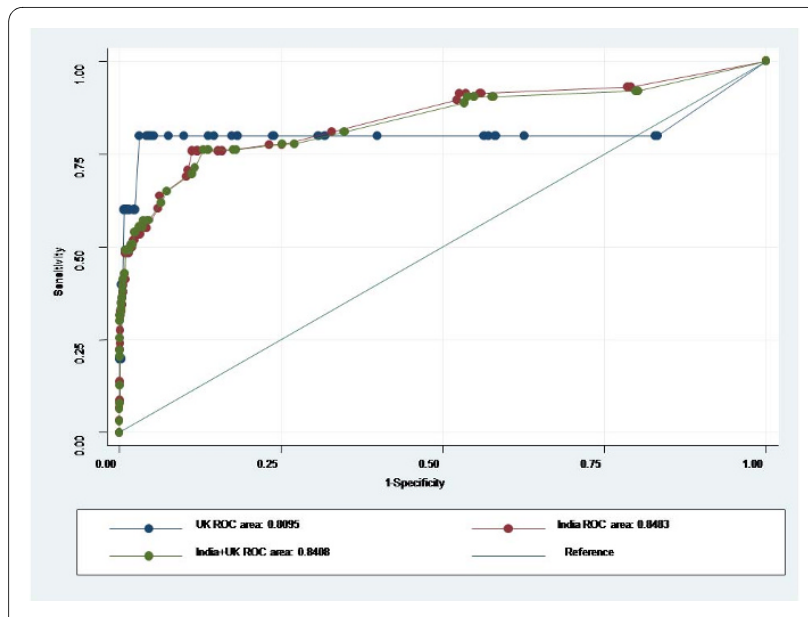

Figure 1 Areas under ROC curves.

therefore not detract from the satisfactory performance shown by the areas under the ROC curves.

The small number of deaths in the UK arm (only 5) explains the shape of its curve. Out of these 5 deaths, one child had a score of zero and the remaining 4 had scores of $\geq 3$. In between these scores $(>0$ and $<3$ ) lay most (nearly $80 \%$ ) of the cohort, where the absence of deaths effectively locked the sensitivity and flattened the curve. A smoother curve would have been expected with a larger number of events (in this context, deaths), which would have been more likely to be spread over different SICK scores.

We have not proposed cutoff scores to categorize patients. In order to do this a database with a large number of events (deaths) would be needed. Where most children presenting to hospital do not die, this would necessitate a huge sample size, certainly much larger than the combined numbers in this study. Furthermore, although cut off scores are necessary for the practical

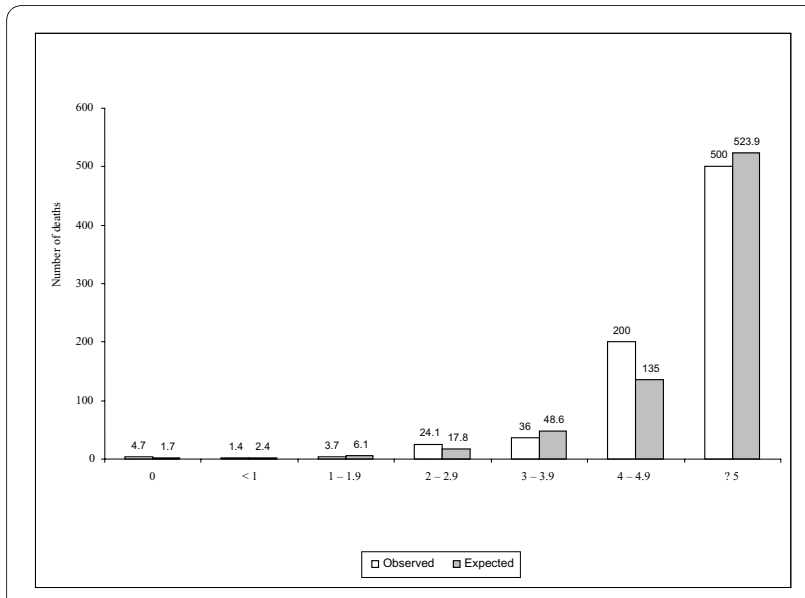

Figure 2 Observed and Expected number of deaths per 1000 in each score category application of a triage system, these will vary in accordance with local conditions. These in turn reflect the balance between risk aversion and the costs and drawbacks of hospital admission in the context of available resources. We tested various cut-off values. The best cutoff for this total data was 1.5 with $77.8 \%$ sensitivity \& $74.8 \%$ specificity. If the cut-off is set at 2 the sensitivity is $76.2 \%$ and specificity is $87.0 \%$ and at 2.5 the sensitivity is $57.1 \%$ and the specificity is $95.5 \%$.

\section{Conclusion}

The SICK score uses only physical criteria without needing recourse to laboratory results. This has cost implications and also makes it immediately determinable on presentation. Furthermore, no special training is needed for its implementation. On this basis we propose it for consideration as a triage tool in resource poor settings.

\section{Competing interests}

The authors declare that they have no competing interests.

\section{Authors' contributions}

MAG, JR, DAG and JP planned this two-centre study. MAG and MS were responsible for the data collection and analysis in Delhi and $\mathrm{AC}$ and $\mathrm{RH}$ for that in London. VS was the statistician for the project and AP developed the computer software - the 'SICK Score Calculator'. MAG, DAG and JP were responsible for the initial draft which was revised with input from the authors. All authors read and approved the final manuscript.

\section{Acknowledgements}

None

\section{Author Details}

'Department of Pediatrics, St Stephens Hospital, Delhi, India, 2 Department of Pediatrics, West Middlesex University Hospital, UK, ${ }^{3}$ GonzoBuzz LLP, 14

Robinson Road, 13-00 Far East Finance Building, Singapore and ${ }^{4}$ Department of Biostatistics, All India Institute of Medical Science, New Delhi, India

Received: 19 August 2009 Accepted: 26 April 2010

Published: 26 April 2010

\section{References}

1. Morgan RJM, Williams F, Wright MM: An early warning scoring system for detecting critical illness. Clin Intens Care 1997, 8:100.

2. Subbe CP, Kruger M, Rutherford P, Gemmell L: Validation of a modified early warning score in medical admissions. Quarterly Journal of Medicine 2001, 94:521-6.

3. Barlow G, Nathwani D, Davey P: The CURB65 pneumonia severity score outperforms generic sepsis and early warning scores in predicting mortality in community-acquired pneumonia. Thorax 2007, 62:253-259. Published Online First: 23 August 2006. doi:10.1136/ thx.2006.067371

4. Yeh TS, Pollack MM, Ruttimann UE, Holbrook PR, Fields A: Validation of a Physiologic Stability Index for Use in Critically III, infants and children. Pediatric Res 1984, 18:445-451.

5. Pollack MM, Ruttimann UE, Getson PR: The Pediatric risk of mortality (PRISM) score. Crit Care Med 1988, 16:1110-6.

6. Slater A, Shann F, Pearson G, Paediatric Index of Mortality (PIM) Study Group: PIM 2: a revised version of the Paediatric Index of Mortality. Intensive Care Med 2003, 29:278-85.

7. Grove S, Tamburlini G, Molyneux E, Whitesell P, Campbell H: Development and simplified basis of simplified guidelines for emergency triage assessment and treatment in developing countries. Arch Dis Child 1991, 81:473-7. 
8. Thompson MJ, Coad N, Harnden A, Mayon-White R, Perera R, Mant D: How well do vital signs identify children with serious infections in paediatric emergency care? Arch Dis Child published 2009. 101136/adc.2009.159095

9. Sun D, Aikawa $N$ : The natural history of the systemic inflammatory response syndrome and the evaluation of SIRS criteria as a predictor of severity in patients hospitalized through emergency services. Keio J Med 1999, 48:28-37.

10. Kumar N, Thomas N, Singhal D, Puliyel JM, Sreenivas V: Triage score for severity of illness. Indian Pediatr 2003, 40:204-10.

11. Bhal S, Tygai V, Kumar N, Sreenivas V, Puliyel JM: Signs of Inflammation in Children that can Kill (SICK score): Preliminary prospective validation of a new non-invasive measure of severity of illness. J Postgrad Med 2006, 52:102-5.

12. Mackway-Jones K, Molyneux E, Phillips B, Weeteska S: Advanced Pediatrics Life support. The Practical approach. BMJ Books 2001

13. Lemeshow S, Le Gall JR: Modeling the severity of illness of ICU patients. A systems update. JAMA 1994, 272:1049-55.

14. Craig JV, Lancaster GA, Williamson PR, Smyth RL: Temperature measured at the axilla compared with rectum in children and young people: systematic review. BMJ 2000, 320:1174-8.

doi: $10.1186 / 1824-7288-36-35$

Cite this article as: Gupta et al., Validation of "Signs of Inflammation in Children that Kill" (SICK) score for immediate non-invasive assessment of severity of illness Italian Journal of Pediatrics 2010, 36:35

Submit your next manuscript to BioMed Central and take full advantage of:

- Convenient online submission

- Thorough peer review

- No space constraints or color figure charges

- Immediate publication on acceptance

- Inclusion in PubMed, CAS, Scopus and Google Scholar

- Research which is freely available for redistribution

Submit your manuscript at www.biomedcentral.com/submit
Ciomed Central 